an instrument such as the PSE into Yoruba, for example, recourse has to be made to metaphors in common use such as "the heart is weak", for depression, and "the heart is not at rest", for anxiety. One cannot be sure that these phrases correspond at all precisely with the English words.

Not only are some languages richer than others in the language of emotion: even within cultures members of underprivileged groups may have less welldeveloped abilities to recognize or to express nuances of feeling (as Basil Bernstein has demonstrated in British society).

Dr Leff is dedicated to the use of standardized instruments, accompanied by glossaries and sets of instructions as the best way to enable different observers to recognize and record symptoms in the same way. Usually he remains keenly aware of the difficulties which still remain, but very occasionally he lapses as when, after completing a review of a number of surveys of psychiatric illness in Asian countries he draws attention to the wide differences in the prevalence rates for neurosis, and comments: "We can infer that the very highest rates found in the Asian surveys are likely to represent the most realistic estimates of the true prevalence of the neuroses".

Normally, he would be the first to question the concept of the "true prevalence" of neuroses. He knows that minor neurotic symptoms are very common in normal subjects. Determining at what levels of frequency and severity these symptoms should constitute an illness is a judgemental decision. Until some less subjective indicator can be found, the prevalence of neuroses can only be measured in terms of stated, hopefully relevant, operational criteria. As yet there is no such thing as the "true prevalence" of neuroses and perhaps never will be.

The chapters on the mental health of immigrants to the USA and to Britain are interesting not only for their findings (that, in general, immigrants show a high rate of incidence of schizophrenia but that Asian immigrants attend their general practitioners for minor complaints less often than do their British neighbours), but also because they show that useful crosscultural research can now be carried out within the British Isles and USA. One factor, however, appears to have been overlooked. This is Srole et al.'s report, Mental Health in the Metropolis: The Midtown Manhattan Study (McGraw-Hill, 1962), which found very high rates of psychiatric disorders among immigrants but only among poor immigrants. The much smaller number of rich immigrants did not experience an excess of these disorders.

The final chapter tells us, in grim detail, about the many handicaps which an Asian immigrant physician has to contend with in trying to advance his career in a Western country: these are economic, linguistic, social, educational as well as professional. Having taken a special interest in the welfare of overseas postgraduate students at the Institute of Psychiatry, London, Dr Leff knows what he is talking about; but here he seems to present a totally gloomy picture. Every experience of the immigrant doctor is a negative one, culminating for some in a neurotic or psychotic breakdown, with all their disastrous implications. Is there no gleam of light at all in the experience of our immigrant trainees? Surely we can all remember some exceptions, men and women who have earned the friendship and respect of their British or North American colleagues and have embarked upon an academic or clinical career. Admittedly, they are in the minority. This chapter, although tangential to the rest of the book, will serve a useful purpose by reminding us about the difficult lot of our overseas trainees.

G. Morris Carstairs is currently a Fellow of the Woodrow Wilson Center, Washington DC, and recently spent three years in India as a visiting professor of psychiatry.

\section{Art of insects}

\section{J.L. Cloudsley-Thompson}

Insects Etc.: An Anthology of Arthropods Featuring A Bounty of Beetles. Pp.108. ISBN 0-933920-25-3. (Hudson Hills: 1981.) $\$ 50$.

WHAT is there in common between Edgar Allan Poe, Jonathan Swift and Raoul Hausmann? The answer, immediately revealed by perusal of the literary anthology with which P.A. Gette enlivens this unusual book, is that they were not good observers of insects. Like Salvador Dali's pre-1950 ants, Hausmann's houseflies had only four legs, Swift's were endowed with stings and Poe confused bugs with beetles. Far more observant were Lewis Carroll and Vladimir Nabokov and, of course, Réaumur, Thoreau and JeanHenri Fabre. Buffon was evidently a careless writer: "The insect is a small animal without blood. The latter [sic] can be divided into large and small kinds".

Literary activities, like art, are not necessarily marred by inaccuracy such as this. Less excusable, however, are the errors of nomenclature and spelling apparent in the names of the various arthropods portrayed. For instance, Argiope bruennichi (mis-spelled) is an impressive, striped, orb-web spinner, but it is not the zebra spider. This name has always been reserved for Salticus scenicus the little jumping spider, common on walls and fences throughout Europe, Asia and America. Again, the scorpion is not Buthus occitanus as stated but, unmistakably, Scorpio maurus, the classical "scorpio" of antiquity.

The 34 dazzling, large-format colour plates are taken from photo-realistic paintings by Bernard Durin, without doubt an unusually gifted illustrator in the great tradition of Audubon, Edward Lear and Louis Agassiz Fluertes. Of 33 species illustrated (there is a close-up of the head of Zonocerus variegatus in addition to a painting of the whole grasshopper), 16 are beetles, 5 grasshoppers, 6 Hemiptera, 3 Hymenoptera, a single butterfly (the peacock), a spider and a scorpion. The species depicted are, as might be expected, large, brightly coloured and sometimes bizarre. The paintings illustrating them are breathtaking. Durin is not only a meticulous observer but he really succeeds in creating the illusion of irridescence in his beetles and in making insects' wings look transparent. A commentary on each of the arthropods illustrated, with information about various aspects of its behaviour, life cycle, food, enemies and folklore, is provided by Gerhard Scherer.

Zoologists are often so familiar with the subjects of their research that they overlook obvious questions posed by them. What are the functions of the extraordinary colours of some insects, why do others have such long legs or antennae, what are the advantages and drawbacks of their shapes and sizes? It might be worthwhile taking a second, thoughtful look at the subjects of Durin's paintings.

J.L. Cloudsley-Thompson is Professor of Zoology at Birkbeck College, University of London.

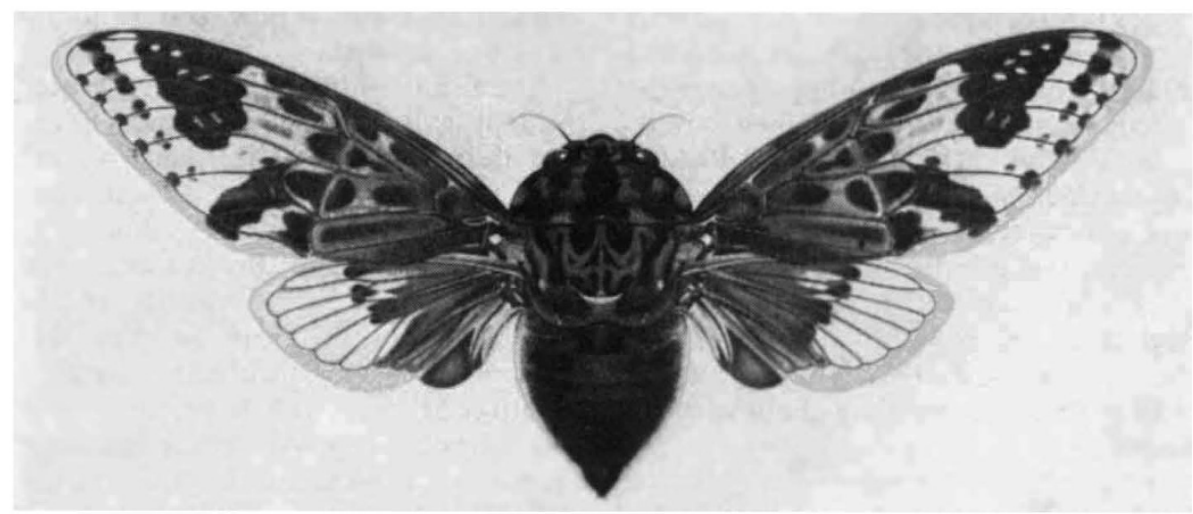

Pycna antinorii, the African cicada, painted by Bernard Durin. Reproduction in black and white.and reduction in size do scant justice to the picture. 